Jianlin Liu*, Runni Wu, and Re Xia*

\title{
Surface effects at the nanoscale based on Gurtin's theory: a review
}

\begin{abstract}
The fields of nanotechnology and nanoscience are full of opportunities and challenges. The needed modification of classical continuum mechanics to account for the dramatically novel characteristics and phenomena determining the mechanical response of nanomaterials/ structures remains an ambitious goal pursued by mechanics researchers. The theory of surface elasticity proposed by Gurtin and Murdoch has been shown to be an important tool in theoretical nanomechanics. In this paper, we present an overview of recent advances in application of surface elasticity theory at the nanoscale. In particular, we focus on the elastic and plastic deformation, vibration and buckling, fracture and contact behavior of nanoscale solids from one dimension to three dimensions. We hope that this contribution can provide a valuable insight into nanomechanics analysis methods by taking surface effects into account. The results may help to bridge the gap between conventional mechanics and findings from simulation and experiment, in such areas as multifunctional material and micro-electro-mechanical systems.
\end{abstract}

Keywords: nanomaterials; nanomechanics; nanostructures; size effect; surface effects; surface elasticity; surface stress.

DOI 10.1515/jmbm-2014-0016

\section{Introduction}

With the rapid development of nanoscience and nanotechnology, more and more studies have focused on capturing the basic physical and chemical characteristics of nanomaterials, which can provide theoretical support

*Corresponding authors: Jianlin Liu, Department of Engineering Mechanics, China University of Petroleum, Qingdao 266580, China, e-mail: liujianlin@upc.edu.cn; and Re Xia, School of Power and Mechanical Engineering, Wuhan University, Wuhan, 430072, China; and Nanoscience Centre, University of Cambridge, $11 \mathrm{~J}$.J. Thompson Avenue, Cambridge CB3 OFF, UK, e-mail: xiare@whu.edu.cn Runni Wu: School of Power and Mechanical Engineering, Wuhan University, Wuhan, 430072, China and experimental verification for improving the manufacturing processes of nanodevice and the technical means of nanomeasurements [1-4]. Without doubt, in many fields such as catalysis and fuel cell, drug delivery and micro-electro-mechanical systems (MEMS), a lot of nanoresearch conclusions are promoting the reformation of conventional laws based on a macroscopic view [5-7]. Some noteworthy achievements which help to clarify the novel mechanical phenomena at the nanoscale, were beyond the boundaries of validity of classical mechanics. The difference of mechanical properties between macroscopic and nanoscopic configurations appears not only in one-dimensional (1D) but also in complex threedimensional (3D) structures. This is called a bottom-up distinction, which covers a lot of branches in mechanics, e.g., solid mechanics, fluid mechanics, rheology, tribology and biomechanics [8-10]. Consider the case of the finite element method (FEM), e.g., although a very useful method of analysis in computational mechanics, it seems to be unable to operate at the nanoscale area and, instead, molecular dynamics (MD) simulation is used. This is based on solving Newton's equations of motion for a collection of interacting particles over a number of time steps, thus creating a more effective method to investigate the deformation and failure of nanomaterials [11]. Likewise, in nanoscale measurements, new measuring devices such as atomic force microscopes (AFMs) and nanoindenters have already been developed to obtain data on mechanical properties in an enormous number of experimental studies [12, 13]. In solid mechanics, by contrast, the theoretical breakthroughs for interpreting nanoscale phenomena and deriving new results grow at a snail's pace and remain controversial. Nevertheless, there are considerable achievements that are worthy of being mentioned [14]. In this progress report, recent theoretical studies on the mechanical properties of nanomaterials/structures are briefly reviewed, and new analytical models proposed to explain or predict the basic mechanical deformation of nanoscaled solids are described.

This paper starts with a brief introduction where different types of theory models for nanomechanics are described, e.g., the gradient theory as pioneered and popularized by 
Aifantis and Fleck/Hutchinson, as well as the Gurtin-Murdoch theory of surface elasticity. Next, an outline of this latter theory is presented. Then, specific investigations related to the mechanical behavior of $1 \mathrm{D}$ nanostructures based on surface elasticity theory are presented. They include bending, buckling, vibration and adhesion of beams with different boundary conditions. Recent progress in complex 2D and 3D nanostructures/nanomaterials is also reviewed. Finally, the applicability of surface elasticity theory on fracture and contact mechanics is discussed.

\section{Theoretical models of nanomechanics}

Of all mechanical properties of nanomaterials, the size effect problem has perhaps attracted most attention among researchers in the area of solid mechanics from nanoparticles to nanodevices $[15,16]$. This effect refers to the size-dependent mechanical properties of materials which become appreciable only in the small-size regime, especially at the nanoscale. Roughly speaking, two identical materials with geometrical similarity may exhibit two distinct behaviors because they merely have the different physical dimensions. For example, the yield strength of the gold beam varies several decuples in diameters of $50 \mathrm{~mm}$ and $50 \mathrm{~nm}$ [17]. It is very difficult to explain this phenomenon using the theory of classical mechanics. Thus, the absence of an appropriate theoretical basis to interpret related experimental and computational findings strongly stimulates further explorations for establishing a new mechanics analysis framework at the nanoscale. Over the course of the last 30 years, many theoretical models describing the size-dependent mechanical behavior of small-scaled materials and structures have been developed in a variety of directions. To the best of our knowledge, at least four popular theories are widely available, as are briefly discussed in the following.

First, we refer to the gradient theory of Aifantis for plasticity which is based on enhancing the flow stress and Hooke's law with the Laplacian of the effective plastic strain and the Laplacian of Hookean stress, respectively. The Laplacian emerges by expanding the integral expression, for average quantifies in a Taylor series involving spatial derivatives of the corresponding local quantifies and retaining the first few terms as desired. Several problems were resolved by this theory, including the prediction of shear bandwidths and spacings, as well as the elimination of elastic singularities from dislocation lines and crack tips [18-30]. Another type of strain gradient plasticity theory was developed by Fleck/Hutchinson and co-workers based on the concept of geometrically necessary dislocations (e.g., [31, 32] and references quoted therein). Soon afterward, this theory was advanced by others, e.g., Nix and Gao [33, 34], Huang et al. [35, 36] and others [37, 38], resulting in the establishment of the mechanismbased strain gradient plasticity model or, as it is called, MSG or CMSG. These strain gradient theories have been applied successfully to analyze the size effect observed during micro/nanoindentation experiments, to analyze near-tip fields of cracks, to model size effects in particulate metal-matrix composites [39] and also to consider the size-dependent mechanical behavior of microbeam and microplates [40, 41]. Secondly, we refer to Eringen's generalized continuum models resting on his theory of micropolar elasticity or the micro-continuum field theory [42]. In such micro-continuum theories, the response of a particle with a microvolume may be related to every other point of the continuum and the associated microrotation and microstretch fields. In particular, the microrotations are treated as independent degrees of freedom in addition to the usual linear displacement fields. Accordingly, various models such as micromorphic elasticity and microstretch elasticity are generated as special cases of this general micro-continuum field approach [43, 44]. Related applications cover the micro/nanomechanics of carbon nanotubes, biomechanics of bone, and so on $[45,46]$. Thirdly, we refer to the theory of nonlocal continuum mechanics (nonlocal elasticity) that was formally launched in the literature by Eringen, as well as Eringen and Edelen [47, 48]. The main difference between this theory and the conventional continuum mechanics is that long-range force is incorporated, such that the stress state at a given reference point is a function of the strain field at every other point in the body. This makes it possible to describe the size effect through appropriate constitutive equations involving a characteristic size-dependent material parameter. This theory has been applied successfully to consider the lattice dispersion of elastic waves, fracture mechanics, dislocation mechanics, as well as the mechanical behavior of nanomaterials and nanodevices [49-52]. Finally, we refer to the approach discussed here, that is, the theory of surface elasticity developed by Gurtin and co-workers [53-55]. This theory is also a useful continuum mechanics tool for analyzing size-dependent phenomena, especially those observed at the nanoscale. Recently, this theory has been used for the interpretations and related comparisons of size-dependent results from experiments and atomistic simulations [56-58]. A brief account of the basic ideas and a detailed review on recent progress of surface elasticity theory are presented in the following sections. 


\section{Gurtin-Murdoch theory of surface elasticity}

To some extent, the increasing surface-to-volume ratio is considered to be the main reason for the most distinct differences observed in the mechanical, physical and chemical properties between nanoscopic and macroscopic materials. The higher this ratio is, the more predominant the surface effects will be. The near-surface atoms in a material reside in a local environment different from that in the interior, leading to the emergence of a surface layer (typically with a thickness of $\sim 1 \mathrm{~nm}$ ) having physico-chemo-mechanical properties and energy density distinctly different from its bulk counterpart [59]. As a typical example, we consider in Figure 1 a nanosphere with a very thin surface layer which possesses a very high surface energy. Obviously, the ratio of the surface area to the volume of the sphere is inversely proportional to its radius; therefore, a nanoparticle is expected to exhibit the significant surface effects.

In the theory of surface elasticity, a surface is normally viewed as a two-dimensional heterogeneous thin film attached to its bulk. The fundamental equations in the bulk are identical to those of the classical theory of elasticity, except that the boundary conditions are modified to account for the effect of surface stresses. The surface layer and the bulk material are assumed to be bonded perfectly so that their displacements are continuous across the interface. Thus, the equilibrium equation and its nonclassical boundary conditions can be written as $[58,60]$

$$
\begin{aligned}
\tau_{\alpha \beta, \beta}+t_{\alpha} & =0, \\
\tau_{\alpha \beta} k_{\alpha \beta} & =\sigma_{i j} n_{i} n_{j},
\end{aligned}
$$

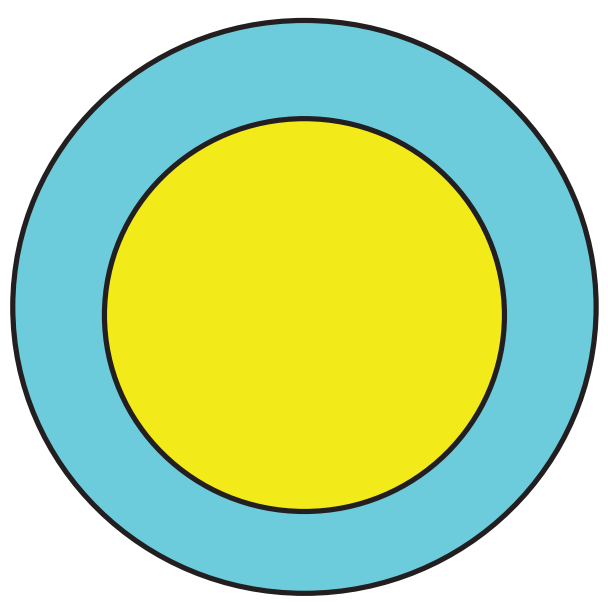

Figure 1 A nano-sphere with a surface layer. respectively, where $n_{i}$ is the unit normal vector of the surface layer, $t_{\alpha}$ is the tangential component of the traction $\sigma_{i j} n_{j}$ along the $\alpha$ direction, $k_{\alpha \beta}$ is the curvature tensor of the surface and $\tau_{\alpha \beta}$ is the surface stress tensor. [Throughout this paper, Einstein's summation convention is adopted for all repeated Latin indices $(1,2,3)$ and Greek indices $(1,2)$.]

It can be seen from Eq. (1) that the volume stress has a jump at the surface but the condition of displacement continuity holds true. In an Eulerian framework, the surface stress tensor $\tau_{\alpha \beta}$ and the surface energy density $\lambda$ satisfy the Shuttleworth equation

$$
\tau_{\alpha \beta}=\lambda \delta_{\alpha \beta}+\frac{\partial \lambda}{\partial \varepsilon_{\alpha \beta}},
$$

where $\varepsilon_{\alpha \beta}$ is the in-plane component of the bulk strain tensor at the surface and $\delta_{\alpha \beta}$ is the Kronecker delta. Equation (2) provides a constitutive relation in two dimensions for the surface. For simplicity, we suppose that both the surface layer and bulk material are isotropic and linearly elastic, and that the surface layer has the uniform thickness $t_{0}$. Hence, the constitutive equation for the surface elasticity theory can be written as

$$
\tau_{\alpha \beta}=\tau_{\alpha \beta}^{0}+S_{\alpha \beta \gamma \delta} \varepsilon_{\gamma \delta},
$$

where $\varepsilon_{\gamma \delta}$ is the surface strain tensor, $S_{\alpha \beta \gamma \delta}$ is the surface elastic constant tensor and $\tau_{\alpha \beta}^{0}$ is the residual surface stress tensor when the bulk is unstrained. Most surfaces (especially those in metallic materials) have anisotropic properties, and they can induce large and systematically anisotropic strain in the bulk of nanoporous materials, as described by Weissmüller et al. [61, 62]. For the sake of simplicity, however, we assume that the surfaces in the nanoporous materials are isotropic, or in others words, they have orientation-averaged elastic properties. In this case, the constitutive relation in Eq. (2) reads [53, 54]

$$
\tau_{\alpha \beta}=\tau_{\alpha \beta}^{0}+2 \mu_{s} \varepsilon_{\alpha \beta}+\lambda_{s} \varepsilon_{\gamma \gamma} \delta_{\alpha \beta},
$$

where $\lambda_{s}$ and $\mu_{s}$ are the surface Lamé constants. The constitutive relation in Eq. (3) can also be written in other forms [63]. When the surface is subjected to uniaxial tension, Eq. (3) can further be simplified as $[58,60]$

$$
\tau=\tau_{0}+E_{s} \varepsilon,
$$

where $\tau$ is the surface stress, $\varepsilon$ is the surface strain and $\tau_{0}$ is the residual surface stress under the bulk unstrained condition. The symbol $E_{s}$ is referred to as the surface 
Young's modulus, which was initially defined by Gurtin and Murdoch [53]. It is related to the surface Lamé constants by $E_{s}=2 \mu_{s}+\lambda_{s}$. Theoretically, the value of $E_{s}$ may be positive or negative, depending on the crystalline structure and the orientation of the surface [55, 64]. For different materials, therefore, the elastic modulus of a nanowire may either increase or decrease with decreasing size.

\section{Surface elasticity theory for 1D nanostructures}

Just like in conventional continuum mechanics, we consider the deformation of an Euler beam as the most typical example, for exploring size effects at the nanoscale. In fact, such 1D nanostructures were first employed to study surface effects on mechanical behavior. In particular, these studies were concerned with the size dependence of the measured elastic modulus of metals and $\mathrm{ZnO}$ nanowires, as well as the calculated bending properties of FCC nanowires $[57,65,66]$. The first well-done implementation of Gurtin's surface elasticity theory at the nanoscale may

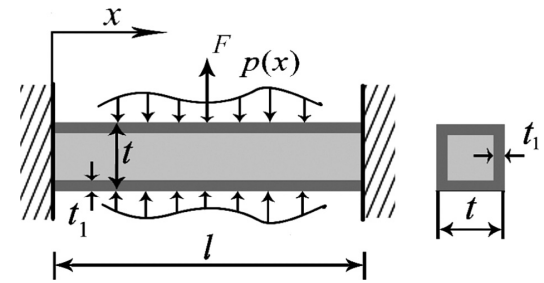

Figure 2 Clamped-clamped square nanobeam subjected to a lateral force $F$. The residual surface stress acts as a distributed load $p(x)[70]$.

their study of Euler-Bernoulli nanobeams via the YoungLaplace equation. Figure 2 shows a double-clamped square nanobeam model incorporating the surface effects with a surface layer, where $t$ denotes the thickness of the nanobeam, $l$ the length and $t_{1}$ the thickness of the surface layer [70]. According to the Young-Laplace equation, a transversely distributed force is produced due to the residual surface stress spanning in the longitudinal direction of the beam. Based upon this model, the explicit solutions for the elastic deformation of a nanobeam with different boundary conditions are obtained, and then, various conclusions associated with different forms of the resulting expressions were provided by Feng et al. [70]:

$$
w(x)=\left\{\begin{array}{l}
\frac{F \cosh (l \eta)}{H_{0} \eta}\left[\eta x-\tanh (l \eta)-\frac{\sinh (\eta x-l \eta)}{\cosh (\eta)}\right], \quad x \in[0, l] \text { (Cantilever) } \\
\frac{F}{2 H_{0}}\left[x-\frac{1}{\eta} \frac{\sinh (\eta x)}{\cosh (l \eta / 2)}\right], \quad x \in\left[0, \frac{l}{2}\right](\text { Sample) } \\
\frac{F}{2 H_{0} \eta}\left[\eta x-\tanh (\eta / 4)-\frac{\sinh (\eta x-l \eta / 4)}{\cosh (\eta / 4)}\right], \quad x \in\left[0, \frac{l}{2}\right] \text { (Double-clamped) }
\end{array}\right.
$$

be traced back to the paper of Miller and Shenoy published in 2000 [58]. They developed a continuum model, taking the surface elastic modulus into account, in order to treat the size effects in tension and bending of nanoplates and nanobars. Compared to related atomistic simulations of nanoscale $\mathrm{Si}$ and $\mathrm{Al}$ structures, the model shows excellent agreement. Such a framework was also expanded and used for the prediction of rigidity of nanosized torsional structures [67]. Soon thereafter, the size-dependent elastic response of nanointerconnects and that of nanoinhomogeneities were considered by Sharma et al. [68] and Duan et al. [69], based on the theory of surface elasticity, by focusing on the nonlocal interactions and on interface stress effects. They concluded that surface elasticity can dominantly influence the fundamental stress state of nanostructures. In this connection, He and Lilley [64] incorporated surface stress and surface elasticity effects in where $w(x)$ is the deflection of the nanobeam at point $x, l$ is the length of the nanobeam and $F$ is the concentrated load. The following notations were used: $H_{0}=2 \tau_{0} t$ and $\eta=\sqrt{H_{0} /(E I)^{\star}}$, where $\tau_{0}$ is the residual surface stress, $t$ is the thickness of the nanobeam and $(E I)^{*}$ is the effective bending stiffness of the nanobeam. When the residual surface stress is neglected, $H_{0}=2 \tau_{0} t=0$, then Eq. (6) becomes consistent with the previous results [64].

Subsequent to the Euler-Bernoulli beam, the elastic response of Timoshenko nanobeams incorporating surface effects was considered. Jiang and Yan [71] and Xia et al. [72] have derived a consistent expression for the bending of a nanobeam with coupling effects between surface stress and shear deformation, by solving the corresponding governing equations and using the principle of minimum potential energy, respectively. The solution is given by 


$$
\bar{w}(x)=\left\{\begin{array}{l}
\frac{F \cosh (l \xi)}{H_{0} V \xi}\left[\left(H_{0}+V\right) \xi x-V \tanh (l \xi)+V \frac{\sinh (l \xi-x \xi)}{\cosh (l \xi)}\right], \quad x \in[0, l] \text { (cantilever) } \\
\frac{F}{2 H_{0} V \xi}\left[\left(H_{0}+V\right) \xi x-V \frac{\sinh (x \xi)}{\cosh (l \xi / 2)}\right], \quad x \in\left[0, \frac{l}{2}\right](\text { Sample), } \\
\frac{F\left[\left(H_{0}+V\right) \xi x-V \tanh \left(\frac{l \xi}{4}\right)+\frac{V \sinh (l \xi / 4-x \xi)}{\cosh (l \xi / 4)}\right]}{2 H_{0} V \xi}, \quad x \in\left[0, \frac{l}{2}\right] \text { (double-clamped) }
\end{array}\right.
$$

where $\xi=1 / \sqrt{(E I)^{\star}\left(1 / H_{0}+1 / V\right)}$ and $V$ is the shear stiffness. Ignoring the effect of shear deformation and letting the shear stiffness approach infinity (i.e., $V=\kappa G A \rightarrow \infty$ ), Eqs. (7) are reduced to the solution for an Euler-Bernoulli nanobeam with surface effects [64].

In particular, there are some reports elaborating on the asymmetric or anisotropic mechanical properties of nanowires. For the asymmetric behavior of tension compression, the surface stress can cause an increase of the tensile yield strength and a decrease of the compressive yield strength, but both the elastic modulus and Poisson's ratio increase as the nanowire radius decreases [73]. Having quantitatively investigated the uniaxial tension deformation of nanoribbons containing antisotropic surface stress effects, Wang et al. [74] demonstrated that anisotropic surface stress may induce a helical or twisting shape of 1D nanostructures.

Considering other types of elastic behavior, a number of papers have attempted to develop modified models with surface effects for vibration and buckling of 1D nanostructures. Mcfarland et al. [75] established a surface-stresscoupled model by which the bending resonant frequency of the microcantilever can be predicted rationally and measured in a simpler manner. Successive efforts by Wang and Feng have been devoted to investigating the effects of both surface elasticity and residual surface tension on the natural frequencies of a microbeam, the surface effects on the transverse vibration of a Timoshenko nanobeam, as well as the influence of surface stress on the vibration frequency of piezoelectric nanowires [60, 76, 77]. Similar analyses as in [60] were done by $\mathrm{He}$ and Lilley [78] to obtain solutions for the resonance frequencies of bending nanowires under different boundary conditions. Most of these results showed that both residual surface tension and surface elasticity may significantly affect the vibration frequency of 1D nanostructures. More specifically, having a positive surface stress will lead to a decrease in the resonance frequency of cantilever nanobeams and an increase in simply supported and clamped-clamped nanobeams. As a complement, Zener's an elastic model was applied to considering the dissipation mechanism together with surface elasticity; thus, the effect of sizedependent surface dissipation on the natural frequency of nanowires was formulated [79]. Resting on the Gurtin-Murdoch continuum theory, Liu and Rajapakse [80] performed a numerical analysis to check the static and vibrational response of both thin and thick nanobeams. Besides the different boundary conditions used, two types of loadings (concentrated and uniformly distributed) were selectively introduced. In the case of nanobeam buckling, several results were obtained between 2007 and 2010 . These suggest that under compression, a positive surface elastic modulus may lead to surface buckling, whereas a negative surface elastic modulus to wrinkles, irrespective of the sign of the surface strain [81]. Moreover, the influences of surface elasticity and residual surface tension on nanobeams are more prominent with increasing slenderness ratio [82]. Also, it was concluded that both the surface effects and the shear deformation should be taken into account for a stubby nanowire so that more accuracy can be secured in related nanomeasurements by microbeam-based methods [76]. Finally, it was found that the residual surface stress and elastic constants of piezoelectric nanowire could be determined by measuring the critical buckling electric potential [77]. By analyzing the postbuckling behavior of a nanowire under compression, it can be deduced that both surface elasticity and residual surface tension are negligible to postbuckling properties but are key to critical loading calculations [83]. Additionally, it must be pointed out that depending on the type of boundary conditions used, nanobeams may indicate whether surface stress will cause a stiffening or softening effect. In other words, the clamped-free boundary condition corresponds to a decrease in buckling load compared to the one without surface effects, whereas hingedhinged and double-clamped boundary conditions induce an increase [84]. Recently, the large displacement of a nanowire under a static bending nanowire with surface effects was considered by using "shooting" methods to obtain numerical solutions of the corresponding boundary value problem [85]. The results on quantitative comparisons between large and infinitesimal deformations 
show that the bending response of nanobeams within a large deformation theory seem to be much "stiffer" than that deduced from a small deformation theory, as shown in Figure 3, where a cantilever beam is selected as an example. This kind of study was extended to the solidliquid interaction; Liu and co-workers also considered the effects of surface elasticity and residual surface stress on the behavior of a nanowires adhered to a substrate [86], as schematically shown in Figure 4. They suggested that the adhesion energy is quite sensitive to the detachment length, so that surface effects could not be neglected in the description of nanoscale adhesion problems.

Meanwhile, computational simulations related to surface elasticity theory were developed. Through such atomistic simulations, Shenoy advanced a model with embedded atom potentials to determine the surface elastic properties of FCC metals [87]. The simulated surface elastic constants obtained in this paper have been used as a reference point for quantitative calculations of nanostructure deformation in many studies on surface effects $[64,70,72]$. By viewing surface stress and surface elastic constant as scalars, Zhang et al. [88] obtained elastic con-

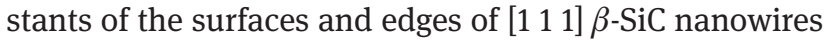

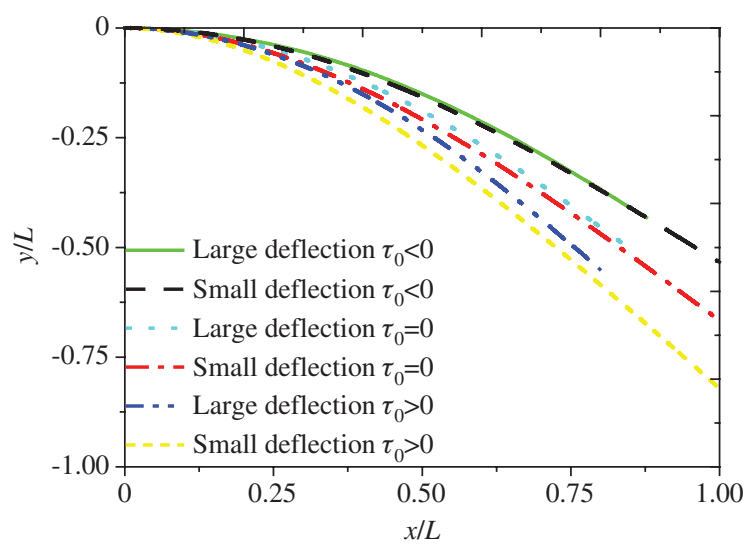

Figure 3 Deflection curves of a cantilever nanobeam in finite or infinitesimal deformation, with or without a surface effect [85].

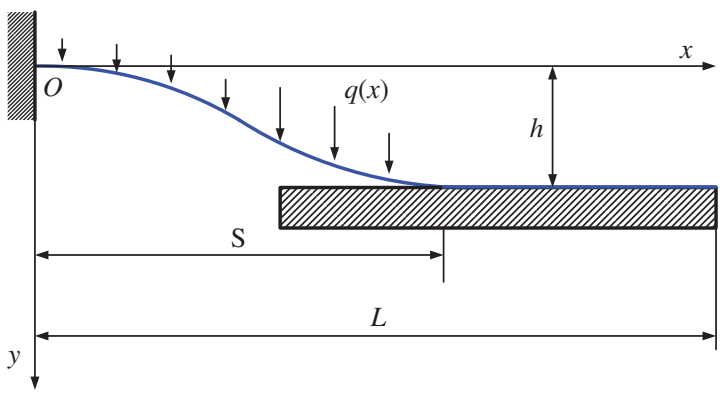

Figure 4 Schematic of a nanobeam adhered on a solid substrate [86]. at freestanding equilibrium and uniaxial strain states through a fundamental energy-based mechanics analysis, in agreement with related MD simulations. Also, the classical FEM was improved to deal with the size-dependent mechanical behavior of nanostructures. Gao et al. [89] developed a special type of surface element to take into account the surface effects. The modified FEM analysis reveals that surface elasticity also plays an important role in the interaction between two pressurized nanovoids and on the elastic properties of 2D nanoporous structures. In addition, Yun and Park [66], using a single-crystal gold nanowire as a sample, performed a 3D nonlinear FEM simulation in which the surface stress was quantified through the Cauchy-Born model, thus producing a new rich harvest on surface effect studies. For instance, one of their conclusions indicates that not only the boundary condition but also the geometry factor of nanowire should be taken into account while analyzing the final stiffening/ softening effects due to surface stress. This brings a new element into the previous view of nanobeam softening through a negative surface stiffness.

\section{Surface elasticity theory on complex nanostructures and nanomaterials}

Nanoscaled complex materials and structures such as bilayer nanofilms, nanocomposites, nanoinclusions and nanoporous materials hold new potential applications in science and engineering. These include sensors and actuators, chemical catalysis, tissue engineering and MEMS/ NEMS devices [90]. This section reviews surface elasticity theory for nanostructures or nanomaterials, by extending it from 1D objects to more complex or composite structures and materials.

Let us consider the application of surface elasticity to 2D nanostructures. The analysis of diffraction of plane harmonic compressional waves yields surface energy having a great influence on the diffraction of an elastic wave around a hole when its radius reduces to nanometers [91]. In a related study of Zang and Liu [92], the Timoshenko model was used to calculate the bending curvature of a strained bilayer nanofilm. The importance of surface stress effects was illustrated by employing the energy minimization method within the framework of continuum mechanics. The finding of Lü et al. [93] on the occurrence of jump-in instability in a nanoplate suggests that surface elasticity can affect the critical separation gap but not the contact length between the ultrathin 
film and substrate. Another of their publications reported that the surface effect will become more significant and indispensable when the thickness of functionally graded films reduces to the intrinsic length scale [94]. Furthermore, an analytical study was conducted to consider not only the action of surface elasticity and residual surface stress but also surface piezoelectricity on the wrinkling of piezoelectric nanofilm [95]. Most recently, Chen et al. [96] compared the shear modulus of nanohoneycombs under various surface effect considerations, i.e., without surface effects, with only surface elasticity and with both surface elasticity and surface residual stress. They concluded that the regular hexagonal honeycomb should not be considered as isotropic but as orthotropic and the discrepancy of elastic moduli in two directions is more obvious with the increase of the cell-wall thickness. By analyzing the in-plane buckling and bending collapses in such nanohoneycomb structures under uniaxial loading, Chen and Pugno [97] found that the failure transition in nanohoneycombs is governed by the width-to-length ratio and that the surface effects become important only when the crosssection size is beyond $100 \mathrm{~nm}$. Most recently, they also established a link between the ratio of stiffness or strength to density and the mechanical properties of nanohoneycomb materials with structural hierarchy [98].

Early in 2004, Sharma and Ganti [99] revisited the classical inclusion model via surface elasticity and built a sizedependent Eshelby tensor for embedded nanoinclusion, including the factor of surface/interface stress, tension, and energies. Then, the modified formulation was applied to discuss three kinds of size-dependent problems: stress concentration at a spherical nanovoid, overall properties of nanocomposites, as well as strain and emission wavelength in quantum dots. Almost within 1 year afterward, Lim and co-workers $[100,101]$ presented two related papers in both of which the theoretical derivation proceeds along Gurtin's theory of surface elasticity. Two problems were solved: one concerned with the effects of surface stress on stress concentration around a nanoscale spherical cavity, and the other concerned with the influence of interface stress on the elastic field inside a nanoscale spherical inclusion. It should be noted that Zhou et al. [102] provided an overview concerning recent works of inclusions in materials, and thus, more details about surface effects on nanoscale inclusions can be found in this review. Duan and co-workers [103-106] conducted a series of nanocomposite material investigations between 2005 and 2007. They employed interface models to study the stress fields of spherical and circular inhomogeneities, the description of elastic constants of heterogeneous solids containing nanoinhomogeneities and the behavior of multiphase composites containing spherical particles or cylindrical fibers with interface stress, as well as to develop effective scaling equations for nanostructures. A related overview was also published in 2009 by these authors [107] on surface elasticity and nanoscale applications. In the same year, another review on the surface energy of nanosturctures with negative curvature was presented by Ouyang et al. [59].

Three-dimensional nanomaterials such as nanoporous materials have attracted wide interest in recent years due to their potential use in the fields of actuators, sensors, catalysis and fuel cells. In particular, their size-dependent mechanical behavior has attained much attention in the last 10 years [108-111]. It was found that surface stress can significantly influence the elastic constants of 3D nanoporous solids, similar to the case discussed earlier for 1D and 2D nanomaterials. By introducing surface elasticity into generalized self-consistent methods, Duan et al. [112] showed that nanoporous materials can be stiffer than their bulk counterparts by surface modification. This suggests a first step to improve the stiffness of nanoporous materials by means of surface modification. Zhang and Wang [113] were the first to study the effect of surface energy on the yield strength of nanoporous materials. It was concluded that the surface has a great effect on the overall yield strength of nanomaterials as well as on the elastic constants. In 2008, two reports about nanosized spherical cavities were published. They concluded that the stress field and stress concentration near a spherical nanocavity are strongly dependent on the residual surface tension, shape and size of the nanocavity, and that there are also several distinct differences on the action of surface effects on the diffraction of the S-wave and P-wave [114, 115]. Early this year, the multiple scattering of vertical shear waves by an array of cylindrical nanoholes was expressed with the aid of displacement potentials and the wave function expansion method with the framework of surface elasticity [116]. It can be seen that the surface effects lower the dynamic stress concentration which also depends on the separation distance between the holes. Ouyang et al. [117] determined quantitatively the stiffening effect of a cylindrical nanohole arraying by using a thermodynamics method. The accretion of strain energy density associated with lattice relaxation and the dangling bond density of atoms is understood to be the major cause of enlarging the inner-surface free energy that leads to the stiffening effect. Next, several explorations were started by employing "structural" type analyses of nanoporous materials through a bottom-up approach. Invoking the classical Gibson-Ashby theory for 3D cellular solids, Feng et al. [70] built a unit-cell micromechanics model to predict the effective Young's modulus of open-cell nanoporous 
material. Figure 5 gives the schematics of the unit cell of a nanoporous material. Xia et al. [72] used the refined Timoshenko beam model to describe the elastic tension and buckling of nanoporous materials with high relative density. Almost immediately afterward, Lu et al. [118] gave a similar discussion, but the cellular configuration used in their investigation is based on the Kelvin open-cell foam model. Furthermore, by using Lakes' framework for materials with structural hierarchy, Xia and co-workers $[119,120]$ extended their results to the elastic deformation of nanoporous materials with hierarchical architectures. The surface effects can increase their plastic strength as well as enlarge their elastic modulus [121]. The studies mentioned above suggest a promising way to design novel porous materials, such as multifunctional foams, porous metamaterials and biomedical implants.

\section{Surface elasticity theory on fracture and contact mechanics}

Surface effects on the fracture analysis of nanomaterials is also a hot research topic nowadays. Wang and co-workers
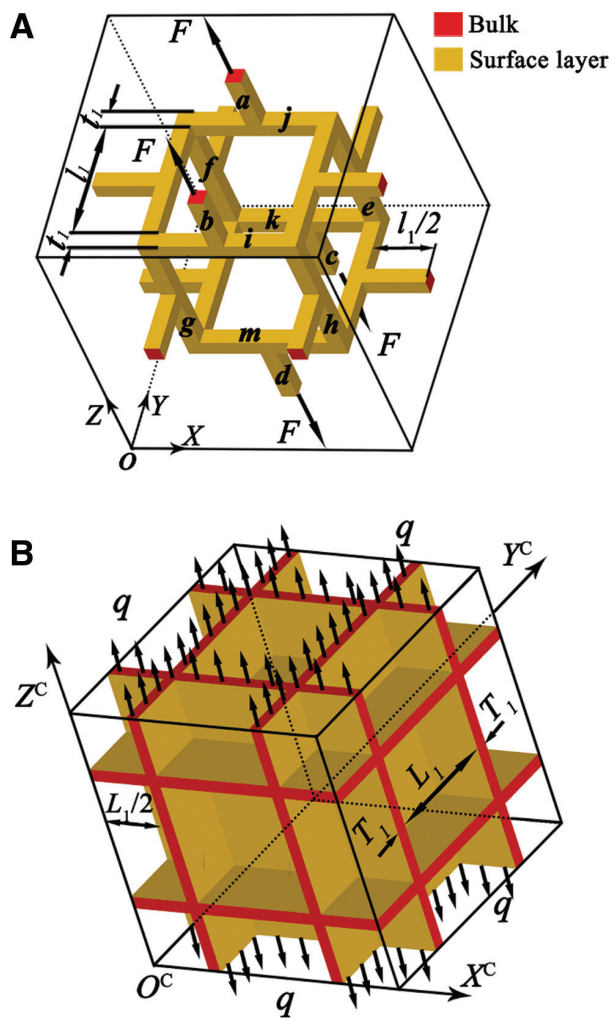

Figure 5 Cubic unit cell model for nanoporous materials with a surface effect: (A) open cell model; (B) closed cell model [119].
$[122,123]$ conducted a number of theoretical and numerical analyses to explore the influence of surface energy on crack tip fields. In the two papers, they considered mode-I, mode-II and mode-III cracks as example cases and used the surface elasticity theory and local asymptotic approach to determine the relation between surface energy and stress distribution near a crack tip with a finite root radius. The results of the theoretical analysis and the corresponding FEM simulation provide the variation of the action of surface energy on different crack modes. It alters the stress magnitudes at the crack tip of the mode-I crack, as well as both the magnitude and position of the maximum stress. For mode-III, it only affects the stress at a small distance ahead of the crack tip. In their follow-up works of FEM simulations, the $J$-integral and elastic field around surface defects were discussed by introducing a kind of surface element to reflect the action of surface energy [124, 125]. As a typical example, the influence of the surface energy and crack root bluntness on the stress distribution near the crack tip is shown in Figure 6 [124]. Lately, other fracture characterization parameters such as the crack-opening displacement and stress-intensity factor were also obtained by Nan and Wang [126]. The impact of residual surface stress on these parameters involves the whole crack surface rather than the surface near the crack tip. Similarly, based on the Gurtin-Murdoch continuum theory and complex variable method, Luo and Wang analyzed the elastic field of an elliptics nanoinhomogeneity embedded in an infinite matrix under anti-plane shear [127]. Moreover, Kim et al. [128] studied the effect of surface elasticity on an interface crack between two dissimilar linearly elastic isotropic homogeneous materials under plane deformation. Intarit et al. [129] gave analytical solutions through which the shear and opening dislocations, as well as the corresponding internal vertical and horizontal loading, in an elastic half-plane can be numerically described by using the Gurtin-Murdoch model and the Fourier integral transform method.

The last topic in this review is to present a few remarks on contact problems at the nanoscale. As of today, there are relatively few works available about the surface effects on contact mechanics. In 2007, surface stress effects were introduced into a nanoscale contact analysis by using surface elasticity theory to determine the influence that the contact normal stress and deformation gradient may have on the deformed surface. This changes smoothly across the loading boundary, and the maximum normal stress and indentation depth largely depend on the surface stress [130]. The situation may be analogous to the 2D Hertzian contact configuration during the indentation of a rigid cylinder on an elastic half-space, as shown schematically in Figure 7. It can be stated that surface tension is an active 

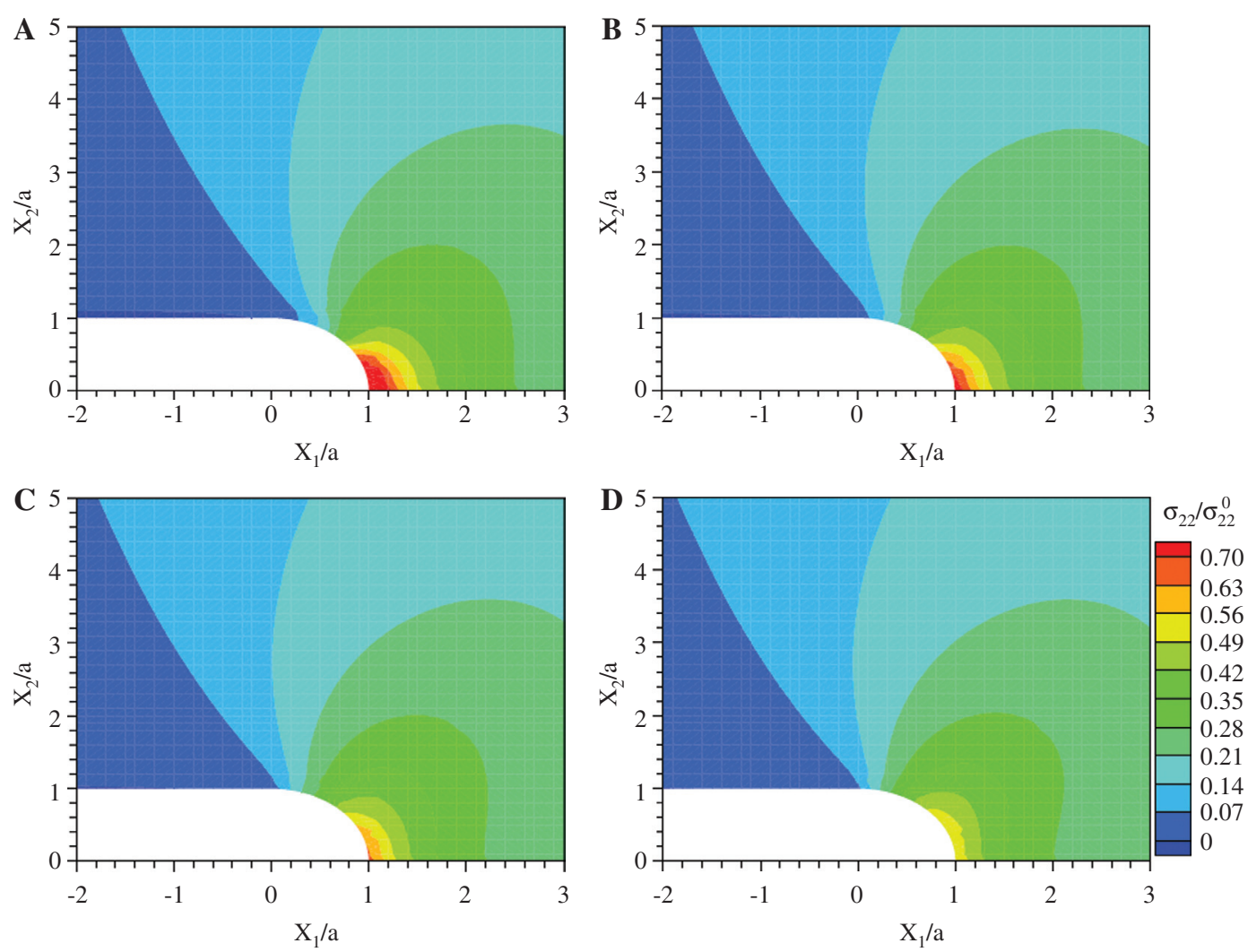

Figure 6 The influence of surface energy and crack root bluntness on the stress distribution near the crack tip [124].

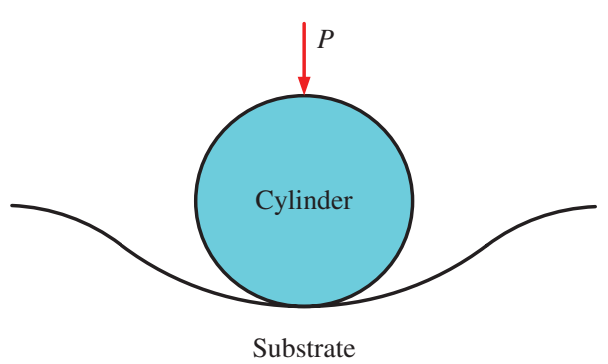

Figure 7 Schematic of the indentation of a rigid cylinder on an elastic half space [131].

participant in determining the pressure distribution in the contact region and the estimated contact width. For sufficiently small contact widths, this could cause a considerable increase in nominal indentation hardness, a result which could be useful for more accurate applications of the nanoindentation technique [131].

\section{Summary and outlook}

Nowadays, researchers are struggling with many puzzling questions arising from the rapidly developing fields of nanotechnology and nanoscience, across a variety of disciplines including domains of material science, thermodynamics, electromagnetics, biology and medicine. In mechanics, classical continuum mechanics is undergoing a massive expansion to face the dramatically novel characteristics and phenomena emerged when considering the mechanical behavior of materials and structures. Considerable progress on theoretical analyses as well as simulations and experiments has been made, and, hence, many sparkling accomplishments have been established. This review only concerns with the applications of Gurtin's theory of surface elasticity to nanoscale mechanical behavior presented in the last decade.

Results from the existing studies have provided a valuable insight into surface effects on the elasticity, plasticity, fracture, and contact behavior of 1D nanostructures, as well as on more elaborate configurations for composites and other complex nanostructures. Based on the Gurtin-Murdoch continuum theory, many conventional physical models and analytical methods are revisited and modified. Such new "weapons" have been successfully applied to explain experimental and simulation findings, and to predict the mechanical properties of material at the nanoscale. It is consistently concluded that surface 
effects (e.g., surface elasticity, surface stress or surface energy) can significantly affect the mechanical behavior at the nanoscale. In other words, as the characteristic size of the object under investigation falls into a small enough range, taking surface factors into consideration in related mechanics analysis of materials and structures is desirable, if not necessary.

Just like in other fields, the development of surface elasticity theories bound by the co-existence of achievement and challenge or, in other words, difficulty and hope go together. The view implied by Wang et al. [132] gives a definite outlook: how to accurately measure and verify the relevance of the surface effects and its related parameters remains a great challenge. That is, what kind of assumptions or what kind of mechanical tests should be used for simplifying the analysis without sacrificing rigor remains an open question. When such questions are answered, the gaps between theory and practice will be bridged with direct implications on material/nanodevice selection/fabrication and utilization.

Finally and once more, three other reviews existing on the topic are highly recommended [59, 102, 132].

Acknowledgments: The authors gratefully acknowledge the supports of the National Natural Science Foundation of China (Grant Nos. 11102140, 11272357 and 11320003), the Doctoral Fund of Ministry of Education of China (Grant No. 20110141120024), the Opening Project of the State Key Laboratory of Nonlinear Mechanics in Chinese Academy of Sciences (LNM201319) and the Science Foundation for Distinguished Young Scholars of Shandong Province (JQ201302). The authors are also grateful to Professor Aifantis for suggesting this task to the first author during a visit of his to Aristotle University of Thessaloniki. The authors are also grateful for Professor Aifantis's comments on an earlier draft of the manuscript.

\section{References}

[1] Lieber CM. MRS Bull. 2003, 28, 486-491.

[2] Hode G. Adv. Mater. 2007, 19, 639-655.

[3] Poole CP, Owens FJ. Introduction to Nanotechnology. Wiley, New Jersey, 2003.

[4] Bhushan B. Springer Handbook of Nanotechnology. Springer, Berlin, 2010.

[5] Ferrari M. Nature Rev. Cancer 2005, 5, 161-171.

[6] Liu H, Song C, Zhang L, Zhang J, Wang H, Wilkinson DP. J. Power Sources 2006, 155, 95-110.

[7] Rossi C, Zhang K, Esteve D, Alphonse P, Tailhades P, Vahlas C. J. Microelectromech. Syst. 2007, 16, 919-931.

[8] Tropea C, Yarin AL, Foss JF. Springer Handbook of Experimental Fluid Mechanics. Springer, Berlin, 2007.
[9] Sharpe WN, Jr, Sharp J, William N. Springer Handbook of Experimental Solid Mechanics. Springer, Berlin, 2008.

[10] Bhushan B. Wear 2005, 259, 1507-1531.

[11] Liu WK, Karpov EG, Park HS. Nano Mechanics and Materials: Theory, Multiscale Methods and Applications. Wiley, New York, 2006.

[12] Withers JR, Aston DE. Adv. Colloid Interface Sci. 2006, 120, 57-67.

[13] Fischer-Cripps AC. Surf. Coat. Tech. 2006, 200, 4153-4165.

[14] Cleland AN. Foundations of Nanomechanics: From Solid-State Theory to Device Applications. Springer, Berlin, 2003.

[15] Roduner E. Chem. Soc. Rev. 2006, 35, 583-592.

[16] Meyers MA, Mishra A, Benson DJ. Prog. Mater. Sci. 2006, 51, 427-556.

[17] Wu B, Heidelberg A, Boland JJ. Nat. Mater. 2005, 4, 525-529.

[18] Aifantis EC. Trans. ASME: J. Eng. Mater. Technol. 1984, 106, 326-330.

[19] Aifantis EC. Int. J. Plasticity 1987, 3, 211-247.

[20] Aifantis EC. Int. J. Eng. Sci. 1992, 30, 1279-1299.

[21] Estrin Y, Kubin LP, Aifantis EC. Scripta Met. Mater. 1993, 29, 1147-1150.

[22] Aifantis EC. Int. J. Eng. Sci. 1995, 33, 2161-2121.

[23] Aifantis EC. Int. J. Non-Linear Mech. 1996, 31, 797-809.

[24] Aifantis EC. Int. J. Fracture 1999, 95, 299-314 [also: Aifantis EC. Strain gradient interpretation of size effects, INV-REVISA(99)P004].

[25] Gutkin MY, Aifantis EC. Scripta Mater. 1999, 40, 559-566.

[26] Aifantis EC. Mech. Mater. 2003, 35, 259-280.

[27] Aifantis EC. Int. J. Eng. Sci. 2009, 47, 1089-1099.

[28] Askes H, Aifantis EC. Int. J. Solids Struct. 2011, 48, 1962-1990.

[29] Aifantis EC. Int. J. Eng. Sci. 2011, 49, 1357-1367.

[30] Walgraef D, Aifantis EC. Int. J. Eng. Sci. 2012, 59, 140-145.

[31] Fleck NA, Hutchinson JW. J. Mech. Phys. Solids 1993, 41, 1825-1857.

[32] Fleck NA, Hutchinson JW. Adv. Appl. Mech. 1997, 33, 295-361.

[33] Nix WD, Gao HJ. J. Mech. Phys. Solids 1998, 46, 411-425.

[34] Gao H, Huang Y, Nix WD, Hutchinson JW. J. Mech. Phys. Solids 1999, 47, 1239-1263.

[35] Huang Y, Gao H, Nix WD, Hutchinson JW. J. Mech. Phys. Solids 2000, 48, 99-128.

[36] Huang Y, Qu S, Hwang KC, Li M, Gao H. Int. J. Plasticity 2004, 20, 753-782.

[37] Chen SH, Wang TC. Int. J. Plasticity 2002, 18, 971-995.

[38] Wei Y, Qiu X, Hwang KC. Eng. Fract. Mech. 2004, 71, 107-125.

[39] Zhu HT, Zbib HM, Aifantis EC. Acta Mech. 1997, 121, 165-176.

[40] Haque MA, Saif MTA. Acta Mater. 2003, 51, 3053-3061.

[41] Huang Y, Zhang F, Hwang KC, Nix WD, Pharr GM, Feng G. J. Mech. Phys. Solids 2006, 54, 1668-1686.

[42] Eringen AC. Microcontinuum Field Theories: Foundations and Solids. Springer: New York, 1999.

[43] Grammenoudis P, Tsakmakis C. Acta Mech. 2007, 189, 151-175.

[44] Lazar M, Maugin GA. Phil. Mag. 2007, 87, 3853-3870.

[45] Rosenberg J, Cimrman R. Math. Comput. Simulat. 2003, 61, 249-260.

[46] Odegard GM, Gates TS, Nicholson LM, Wise KE. Compos. Sci. Technol. 2002, 62, 1869-1880.

[47] Eringen AC. Int. J. Eng. Sci. 1972, 10, 1-16.

[48] Eringen AC, Edelen DGB. Int. J. Eng. Sci. 1972, 10, 233-248.

[49] Peddieson J, Buchanan GR, Mcnitt RP. Int. J. Eng. Sci. 2003, 41, 305-312.

[50] Reddy JN, Pang SD. J. Appl. Phys. 2008, 103, 023511. 
[51] Adali S. Nano Lett. 2009, 9, 1737-1741.

[52] Murmu T, Pradhan SC. Physica E 2009, 41, 1628-1633.

[53] Gurtin ME, Murdoch Al. Arch. Ration. Mech. Anal. 1975, 57, 291-323.

[54] Gurtin ME, Murdoch Al. Arch. Ration. Mech. Anal. 1975, 59, 389-390.

[55] Gurtin ME, Weissmüller J, Larché F. Phil. Mag. A 1998, 78, 1093-1109.

[56] Cuenot S, Frétigny C, Demoustier-Champagne S, Nysten B. Phys. Rev. B 2004, 69, 165410.

[57] Jing GY, Duan HL, Sun XM, Zhang ZS, Xu J, Li YD, Wang JX, Yu DP. Phys. Rev. B 2006, 73, 235409.

[58] Miller RE, Shenoy VB. Nanotechnology 2000, 11, 139-147.

[59] Ouyang G, Wang CX, Yang GW. Chem. Rev. 2009, 109, 42214247.

[60] Wang GF, Feng XQ. Appl. Phys. Lett. 2007, 90, 231904.

[61] Kramer D, Weissmüller J. Surf. Sci. 2007, 601, 3042-3051.

[62] Weissmüller J, Duan HL, Farkas D. Acta Mater. 2010, 58, 1-13.

[63] Ru CQ. Sci. China Phys. Mech. Astron. 2010, 53, 536-544.

[64] He J, Lilley CM. Nano Lett. 2008, 8, 1798-1802.

[65] Chen CQ, Shi Y, Zhang YS, Zhu J, Yan YJ. Phys. Rev. Lett. 2006, 96, 075505.

[66] Yun G, Park HS. Phys. Rev. B 2009, 79, 195421.

[67] Shenoy VB. Int. J. Solids Struct. 2002, 39, 4039-4052.

[68] Sharma P, Ganti S, Ardebili H, Alizadeh A. J. Appl. Phys. 2004, 95, 2763-2769.

[69] Duan HL, Wang J, Huang ZP, Karihaloo BL. J. Mech. Phys. Solids 2005, 53, 1574-1596.

[70] Feng XQ, Xia R, Li X, Li B. Appl. Phys. Lett. 2009, 94, 011916.

[71] Jiang LY, Yan Z. Physica E 2010, 42, 2274-2279.

[72] Xia R, Li X, Qin Q, Liu J, Feng XQ. Nanotechnology 2011, 22, 265714.

[73] Zhang W, Wang T, Chen X. J. Appl. Phys. 2008, 103, 123527.

[74] Wang JS, Feng XQ, Wang GF, Yu SW. Appl. Phys. Lett. 2008, 92, 191901.

[75] Mcfarland AW, Poggi MA, Doyle MJ, Bottomley LA, Colton JS. Appl. Phys. Lett. 2005, 87, 053505.

[76] Wang GF, Feng XQ. J. Phys. D: Appl. Phys. 2009, 42, 155411.

[77] Wang GF, Feng XQ. Europhys. Lett. 2010, 91, 56007.

[78] He J, Lilley CM. Appl. Phys. Lett. 2008, 93, 263108.

[79] Seyyed M, Hasheminejad SM, Gheshlaghi B. Appl. Phys. Lett. 2010, 97, 253103.

[80] Liu C, Rajapakse RKND. IEEE Trans. Nanotechnol. 2010, 9, 422-431.

[81] Wang GF, Feng XQ, Yu SW. Europhys. Lett. 2007, 77, 44002.

[82] Wang GF, Feng XQ. Appl. Phys. Lett. 2009, 94, 141913.

[83] Li Y, Song J, Fang B, Zhang J. J. Phys. D: Appl. Phys. 2011, 44, 425304.

[84] Challamel N, Elishakoff I. Physica E 2012, 44, 1862-1867.

[85] Liu JL, Mei Y, Xia R, Zhu WL. Physica E 2012, 44, 2050-2055.

[86] Liu JL, Xia R, Zhou YT. Chin. Phys. Lett. 2011, 28, 116201.

[87] Shenoy VB. Phys. Rev. B 2005, 71, 094104.

[88] Zhang TY, Luo M, Chan WK. J. Appl. Phys. 2008, 103, 104308.

[89] Gao W, Yu SW, Huang GY. Nanotechnology 2006, 17, 1118-1122.

[90] Lieber CM, Wang ZL. MRS Bull. 2007, 32, 99-108.

[91] Wang GF, Wang TJ, Feng XQ. Appl. Phys. Lett. 2006, 89, 231923.

[92] Zang J, Liu F. Appl. Phys. Lett. 2008, 92, 021905.

[93] Lü CF, Wu D, Chen WQ. IEEE Trans. Nanotechnol. 2011, 10, 962-967.
[94] Lü CF, Chen WQ, Lim CW. Compos. Sci. Technol. 2009, 69, 1124-1130.

[95] Li Y, Fang B, Zhang J, Song J. J. Appl. Phys. 2011, 110, 114303.

[96] Chen Q, Pugno NM, Li Z. Physica E 2013, 53, 217-222.

[97] Chen Q, Pugno NM. Europhys. Lett. 2012, 98, 16005.

[98] Chen Q, Pugno NM. Eur. J. Mech. A 2013, 37, 248-255.

[99] Sharma P, Ganti S. J. Appl. Mech. 2004, 71, 663-671.

[100] Li ZR, Lim CW, He LH. Eur. J. Mech. A 2006, 25, 260-270.

[101] Lim CW, Li ZR, He LH. Int. J. Solids Struct. 2006, 43, 50555065.

[102] Zhou K, Hoh HJ, Wang X, Keer LM, Pang JHL, Song B, Wang QJ. Mech. Mater. 2013, 60, 144-158.

[103] Duan HL, Wang J, Huang ZP, Luo ZY. Mech. Mater. 2005, 37, 723-736.

[104] Duan HL, Yi X, Huang ZP, Wang J. Mech. Mater. 2007, 39, 81-93.

[105] Duan HL, Yi X, Huang ZP, Wang J. Mech. Mater. 2007, 39, 94-103.

[106] Wang J, Duan HL, Huang ZP, Karihaloo BL. Proc. R. Soc. A 2006, 462, 1355-1363.

[107] Duan HL, Wang J, Karihaloo BL. Adv. Appl. Mech. 2009, 42, $1-68$.

[108] Ding Y, Chen M. MRS Bull. 2009, 34, 569-576.

[109] Weissmüller J, Newman RC, Jin HJ, Hodge AM, Kysar JW. MRS Bull. 2009, 34, 577-586.

[110] Tappan BC, Steiner SA, Luther EP. Angew Chem. Int. Ed. Engl. 2010, 49, 4544-4565.

[111] Biener J, Hodge AM, Hayes JR, Volkert CA, Zepeda-Ruiz LA, Hamza AV, Abraham FF. Nano Lett. 2006, 6, 2379-2382.

[112] Duan HL, Wang J, Karihaloo BL, Huang ZP. Acta Mater. 2006, 54, 2983-2990.

[113] Zhang WX, Wang TJ. Appl. Phys. Lett. 2007, 90, 063104.

[114] Ou ZY, Wang GF, Wang TJ. Int. J. Eng. Sci. 2008, 46, 475-485.

[115] Wang GF. J. Appl. Phys. 2008, 103, 053519.

[116] Ru Y, Wang GF, Su LC, Wang TJ. Acta Mechanica 2013, 224, 935-944.

[117] Ouyang G, Yang G, Sun C, Zhu W. Small. 2008, 4, 1359-1362.

[118] Lu Z, Zhang C, Liu Q, Yang Z. J. Phys. D: Appl. Phys. 2011, 44, 395404.

[119] Xia R, Feng XQ, Wang GF. Acta Mater. 2011, 59, 6801-6808.

[120] Wang XS, Xia R. Europhys. Lett. 2010, 92, 16004.

[121] Chen Q, Pugno NM. Europhys. Lett. 2012, 97, 26002.

[122] Wang GF, Feng XQ, Wang TJ, Gao W. J. Appl. Mech. 2008, 75, 011001.

[123] Fu XL, Wang GF, Feng XQ. Int. J. Fracture 2008, 101, 95-106.

[124] Fu XL, Wang GF. Eng. Fract. Mech. 2010, 77, 1048-1057.

[125] Fu XL, Wang GF. Acta Mechanica Solida Sinica 2010, 23, 248-254.

[126] Nan HS, Wang BL. Mech. Res. Commun. 2012, 44, 310-314.

[127] Luo J, Wang X. Eur. J. Mech. A 2009, 28, 926-934.

[128] Kim Cl, Schiavone P, Ru CQ. Proc. R. Soc. A 2011, 467, 3530-3549.

[129] Intarit P, Senjuntichai T, Rajapakse PKND. Eng. Fract. Mech. 2010, 77, 3592-3603.

[130] Wang GF, Feng XQ. J. Appl. Phys. 2007, 101, 013510.

[131] Long JM, Wang GF, Feng XQ, Yu SW. Int. J. Solids Struct. 2012, 49, 1588-1594.

[132] Wang J, Huang Z, Duan H, Yu S, Feng X, Wang G, Zhang W, Wang T. Acta Mechanica Solida Sinica 2011, 24, 52-82. 